\title{
OPTIMISATION OF NAVAL SHIP COMPARTMENT LAYOUT DESIGN USING GENETIC ALGORITHM
}

\author{
Dharani Pragada, Venkata Aditya; \\ Banerjee, Akanistha; \\ Venkataraman, Srinivasan \\ Indian Institute of Technology Delhi
}

\begin{abstract}
An efficient general arrangement is a cornerstone of a good ship design. A big part of the whole general arrangement process is finding an optimized compartment layout. This task is especially tricky since the multiple needs are often conflicting, and it becomes a serious challenge for the ship designers. To aid the ship designers, improved and reliable statistical and computation methods have come to the fore. Genetic algorithms are one of the most widely used methods. Islier's algorithm for the multi-facility layout problem and an improved genetic algorithm for the ship layout design problem are discussed. A new, hybrid genetic algorithm incorporating local search technique to further the improved genetic algorithm's practicality is proposed. Further comparisons are drawn between these algorithms based on a test case layout. Finally, the developed hybrid algorithm is implemented on a section of an actual ship, and the findings are presented.
\end{abstract}

Keywords: Optimisation, Early design phases, Decision making, Genetic Algorithm, Ship Layout

\section{Contact:}

Dharani Pragada, Venkata Aditya

Indian Institute of Technology Delhi

Department of Design

India

ddz198405@design.iitd.ac.in 


\section{INTRODUCTION}

Naval ships evolved as platforms and were predominantly utilized by nations to extend their military dominance in seas. The offensive role of warships included the movement of troops over water to seize and control coastal regions on foreign soil while the defensive roles aimed at protecting the merchant fleet. The role of naval ships has evolved with time, and the current naval ships are also seen as diplomatic ambassadors of a nation to provide disaster relief and humanitarian aid.

Traditionally, naval ships were designed to be faster and sturdier while carrying large weapons. Historically the effectiveness of warships was based on the quantity carried and its range. This resulted in the production of larger ships with bigger weapons. However, modern warships are defined as weapon carrying platforms that can evade detection. The need for avoiding detection while in operation has led to the advent of stealth warships, which revolutionised the naval ship design philosophy leading to the migration of naval ship design from visual intimidation to concealed positioning of weapons and sensors to avoid detection while in operation. This shift made modern warships volume centric platforms where despite the ever-increasing size of platforms there exists a shortage of net volume available for crew and personnel on warships. Consequently, there is a need for generating optimized layouts to improve the efficiency of warships and to ensure optimum use of the available volume of space.

Optimization of naval ship layout overtly relies on the expertise of naval architects/ designers. However, as the complexity of modern warships increases the number of parameters needed for the development of an optimised layout increases exponentially, resulting in the need for optimisation tools to assist naval architects/designers. One of the widely used means to solving an optimisation problem is genetic algorithms. In this paper, the authors, assess the feasibility of using various genetic algorithms for optimising layouts of naval ships and undertake the necessary customization of these algorithms to suit requirements of layout of naval ships. It is expected that this will improve the effective utilisation of net space available.

\section{LITERATURE SURVEY}

\subsection{Genetic Algorithms}

Genetic algorithms (GA) are numerical optimisation algorithms inspired by Charles Darwin's theory of natural evolution and nature's way of selection. The most significant advantage of GA is its simplicity and applicability to a large variety of problems without the need for complex mathematical equations and impenetrable algorithms. A typical GA consists of the development of a mathematical equation to represent any optimization requirement and identify the most optimum solution.

\subsection{General Arrangement}

Ship layouts are typically termed as General Arrangement by naval designers. The general arrangement of a ship is an embodiment of all functions a naval architect needs to achieve through the design (Watson, 1998). General arrangement design involves the positioning of a multitude of compartments often involving conflicting inter-compartment relationships and location-specific constraints. This exponentially complicates the development of an optimum naval ship compartment layout and makes layouts designer-specific in nature. The task of a naval designer developing a naval ship layout can be crudely simplified as an act of successfully solving or making trade-offs among conflicting requirements to develop suitable layouts. Therefore, the efficiency and efficacy of layouts developed are highly dependent on the expertise of naval designers.

\section{GENETIC ALGORITHM BASED GENERAL ARRANGEMENT}

The aim of optimisation of the general arrangement of a ship is the development of objectively configured layouts best suited to the demands of a modern warship, affording naval architects an informed choice during the early stages of ship design. However, as best described by Gillespie (2012) the early-stage design of naval ships is characterised by the need to make design decisions in an environment where little information is available. Further, the general arrangement of a modern warship is critically affected by a multitude of factors. Some of the governing parameters include:

1. Minimising inter-compartment movement to facilitate the quick response of crew in emergencies. 
2. Maximising adjacency of functionally related compartment to facilitate operation.

3. Building simulation-based optimization for energy efficiency

4. Survivability based optimization.

5. Maximising functionality by strategic positioning of offensive and defensive equipment.

GA-based optimisation process is known to provide solutions tending to a definitive answer and is highly effective for solving unstructured problems such as naval ship layout optimisation and will aid a trained naval designer in optimising the layouts given the multiple parameters. The present problem is a multi-objective problem and gives rise to a set of pareto-optimal solutions.

A full factorial design with Pareto optimization using the NSGA-II algorithm for building simulationbased optimization is ideal for analysing the trade-offs between the objective functions to choose an optimal solution for unstructured problems like the general arrangement of ships. However, as indicated above, the critical parameters influencing the general arrangement of warships are varied in nature and the procedure can become highly complicated and computationally intensive. So, the authors consider it prudent to segregate the layout optimization of large facilities like the general arrangement of naval combatants into Sequential Optimization steps to facilitate the designer during the early stages of naval ship design as indicated in (Parsons et al., 2008) and (Igrec et al., 2019). The sequence of optimization operation considered apt by the authors of this paper is:

1. Sequence-I: Longitudinal sectioning of a ship and optimum allocation of cabins to these sections for building simulation-based optimization to ensure the energy efficiency of naval platforms and minimizing the influence of ship motions in critical compartments.

2. Sequence-II: Optimum position of compartments in these selected sections to maximize the stability of naval platforms.

3. Sequence-III: Optimization of compartments in a single deck in these sections to minimize inter compartment distance and maximise adjacency of functionally related compartments.

This paper focuses on Sequence-III of the overall general arrangement optimization of naval ships where the cabins to be positioned in a section are fixed and their positioning is to be finalised. One of the earliest objective functions developed for optimization of naval ship layouts predominantly focused on minimising the inter-compartment movement and maximising the adjacency requirement. (Lee et al., 2002). For explanation, this paper uses the same sample layout as shown in Fig 1.

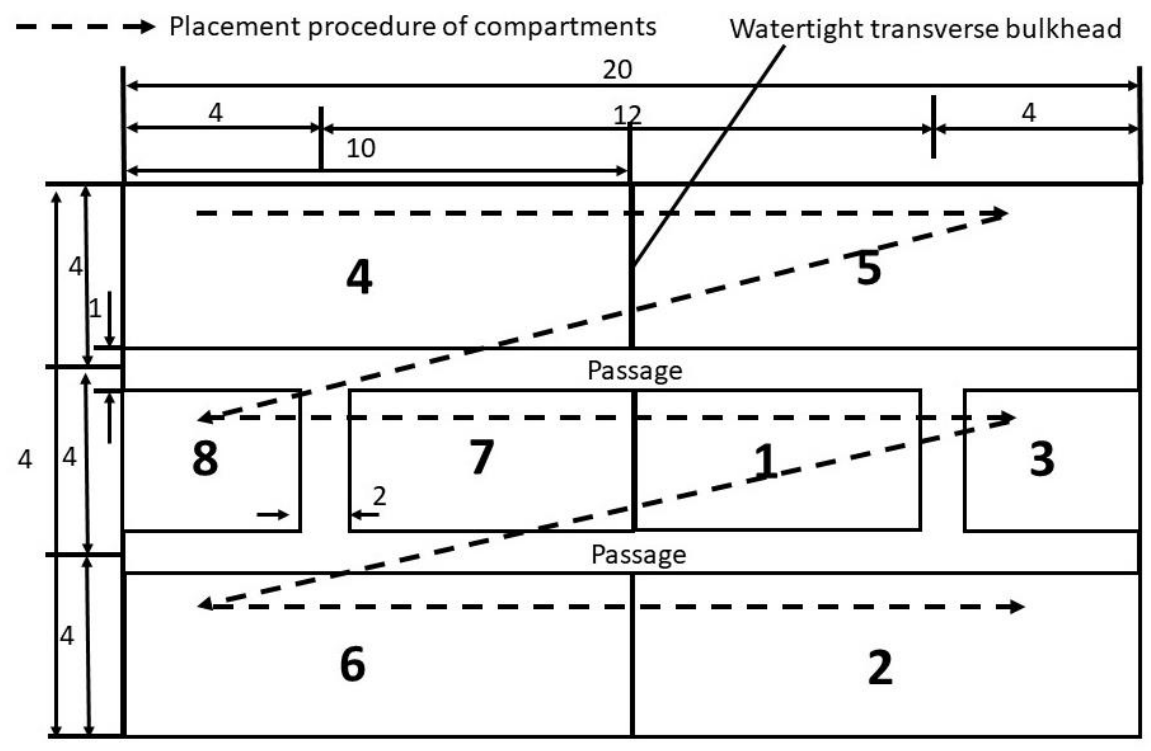

Figure 1. Sample compartment layout (Lee, et al., July 2002)

\subsection{Basic Genetic Algorithm}

The initial layout as shown in Fig 1 has two horizontal and two vertical passageways with fixed locations in the section. The sequence of compartments for the purpose of the program is in zig-zag order from left to right, as shown by dotted lines in Fig. 1. This layout can be encoded for ease of computation as shown in Table 1, where Segment 1 indicates the sequence of the cabins, segment 2 contains the corresponding areas, segment 3 is the distance of the centroid of the horizontal passageways 
from the bottom edge of the layout, and segment 4 is the distance of the centroid of the vertical passageways from the vertical boundaries of the layout.

The optimisation algorithm will aim to reorganise the layout of compartments in Segment 1 while ensuring the requirements of the area of each cabin (Segment 2) and the limitation of arranging the cabins to adhere to the vertical and horizontal boundaries (Segments 3 and 4). The tool considers two random layouts as the initial Parent-1 (P1) and Parent-2 (P2) in the genetic algorithm used. These parent units P1 and P2 are modified using a sequence of operations such as Selection, Crossover, Inversion and Mutation for the individuals of a generation to reproduce into a new generation comprising Children ( $\mathrm{C} 1$ and $\mathrm{C} 2$ ). The resulting fitness function for each of these layouts is calculated to identify the best possible solution satisfying all the constraints in each time frame. Islier (1998) provided some pioneering work in solving multi-facility layout optimisation problems using a genetic algorithm by applying a suitable form of gene encoding and a selection of operators. The drawback of Islier's method is its similarity to conventional optimisation problems such as the Travelling Salesman Problem, where the inter-compartment distance is the shortest distance between their centroids. However, this is not applicable in the case of layout development of multi-functional spaces such as gated community layout, naval ship compartment layout, the layout of a shopping complex where the actual distance covered involves movement from any given compartment to the nearest passageway, traversing along the passageway and movement from the passageway to the required compartment. Lee et al. (2002) improved upon Islier's algorithm for its application in warship compartment layout design by calculating the distance between compartments using Dijkstra's algorithm from graph theory, along with modifying the crossover function into a "modified crossover" operator that was more efficient in minimisation of the objective function and reducing the time taken to convergence.

Table 1. Encoding of sample compartment layout

\begin{tabular}{|c|c|c|c|}
\hline Segment 1 & Segment 2 & Segment 3 & Segment 4 \\
\hline Compartments Numbering & Area of each compartment & Breadth constraint & Length constraint \\
\hline $4,5,8,7,1,3,6,2$ & $35,35,9,15,15,9,35,35$ & $4,4,4$ & $4,12,4$ \\
\hline
\end{tabular}

Dedicated to the Dutch computer scientist Edsger Dijkstra, Dijkstra's algorithm (insert relevant reference) works on the basis that the shortest path from vertices A to E passing through all the vertices involves the need to include the shortest possible sub-paths. In the case of the example shown in Figure 2, the shortest path joining all the vertices is A-B-C-D-E (Barrera, 2020).

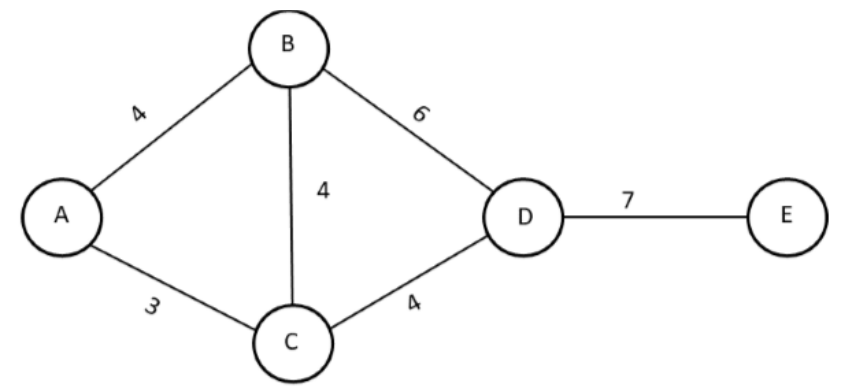

Figure 2. Example of Dijkstra's algorithm

Using Dijkstra's algorithm would physically mean that the movement between compartments only takes place through the passageways. This algorithm was utilised by Lee et al. (2002) for modification of the basic GA to develop an improved genetic algorithm for ship compartment layout design.

\subsection{Improved Genetic Algorithm}

Lee et al. (2002) proposed the Genetic algorithm-based compartment layout of one deck in a naval ship. This work aimed to arrange the various compartments in a specific deck naval ship with different area requirements to minimise the transportation between compartments while catering movement through passageways. The individual objective functions or a mathematical formulation for naval ship layout optimization with the above parameters are as shown below:

Minimize Inter compartment Distance $F_{1}=\sum_{i=1}^{M-1} \sum_{j=1+1}^{M}\left(f_{i j} \times d_{i j}\right)$ 
In the equation above, while $\mathrm{M}$ indicates the number of compartments considered, the variable $\mathrm{d}$ indicates the distance between the compartments in consideration when they are traversed through the passageways while the variable $f$ indicates the expected material flow between the compartments in consideration. While the values of $d$ are calculated using Dijkstra's algorithm, the values of $f$ are defined by the naval designer based on the relation between the compartments.

Maximize Adjacency compliance $F_{2}=\sum_{i=1}^{M-1} \sum_{j=1+1}^{M}\left(b_{i j} \times c_{i j}\right)$

In equation 1(b) also the variable $M$ indicates the total number of compartments considered for evaluation, while the variables $\mathrm{b}$ and $\mathrm{c}$ indicate the inter compartment adjacency factor defined by the designer and adjacency value, respectively.

The equations 1(a) and 1(b) can be unified into a single unconstrained function by the introduction of an external penalty function, which approaches the optimal solution from outside the feasible space in an unconstrained problem. The optimum layout of a deck in a naval ship by minimizing inter compartment distance travelled and maximising the adherence to adjacency of functionally bound compartments based on inputs of the naval architect can be mathematically expressed as an objective function shown below.

Minimize $F=w_{1} \times \sum_{i=1}^{M-1} \sum_{j=1+1}^{M}\left(f_{i j} \times d_{i j}\right)+w_{2} \times \sum_{i=1}^{M-1} \sum_{j=1+1}^{M}\left(\mathrm{C}-b_{i j} \times c_{i j}\right)+\sum\left\{R_{u} \times\right.$ $\left.\max \left(g_{u}, 0\right)\right\}$

In Equation $2\left\{R_{u} * \max \left(g_{u}, 0\right)\right\}$ is the exterior penalty term where $R_{u}$ is the penalty co-efficient and $g_{u}$ is the penalty to be added for the $\mathrm{u}^{\text {th }}$ term. While the values $\mathrm{w} 1$ and $\mathrm{w} 2$ are the weightage given by the designer to the two individual objectives. These weightages afford the designer to objectively accord importance to different objectives.

Table 2. Summary of variables

\begin{tabular}{|c|c|c|}
\hline Variables & Significance & Values \\
\hline $\mathrm{d}$ & Distance & From Dijkstra's algorithm \\
\hline $\mathrm{f}$ & Material flow & Designer input \\
\hline $\mathrm{C}$ & Maximum adjacency rating & 5 \\
\hline $\mathrm{b}$ & Adjacency factor & Values from 0-1 \\
\hline $\mathrm{c}$ & Adjacency value & Values from 0-C \\
\hline $\mathrm{w}_{1}$ & Weight for distance & 50 \\
\hline $\mathrm{w}_{2}$ & Weight for adjacency & \\
\hline
\end{tabular}

The work of Lee et al. (2002) predominantly limited itself to a section of the compartment layout of a ship confined between two boundaries (watertight bulkheads in ship parlance) but suggested the suitability of extending this approach to the layout optimisation of the entire deck of a ship and to multiple decks. Based on the literature review, the improved genetic algorithm is highly efficient in minimising the objective function and arriving at candidate layout solutions. However, the methodology is limited in applicability as it does not consider the limitations imposed by functionality driven compartments like weapon magazine, bridge, machinery compartments that requires a fixed location, size, and aspect ratio. Further, the fixed locations of horizontal and vertical passageways in this approach seemed to be a simplification. These limitations provide an ideal opportunity for an improvement in the layout optimisation tool.

\section{PROPOSED HYBRID GENETIC ALGORITHM}

\subsection{Introduction}

The hybrid genetic algorithm identifies that most sections in a naval ship have dedicated areas allocated to a certain compartment to cater to its float, move and fight requirements. This algorithm attempts to optimise the layout of a section of the naval ship considering the fixed condition of these essential areas. Further, it also attempts to introduce the concept of floating alleyways by considering them as compartments arrangeable within the confines of the layout by adding suitable adjacency requirements. This algorithm also improves on the efficiency of the improved genetic algorithm by employing a local search technique (LST). Mathematically, this means that after each generation it looks for the local 
minima and selects the parents for the next generation from the obtained P1, P2, C1 and C2 based on their relative fitness functions. It aims at maximising the chances of achieving a global minimum at the expense of increased computational time. The process is described schematically in Figure 3. This approach attempts to introduce the concept of modularity in the layout of compartments.

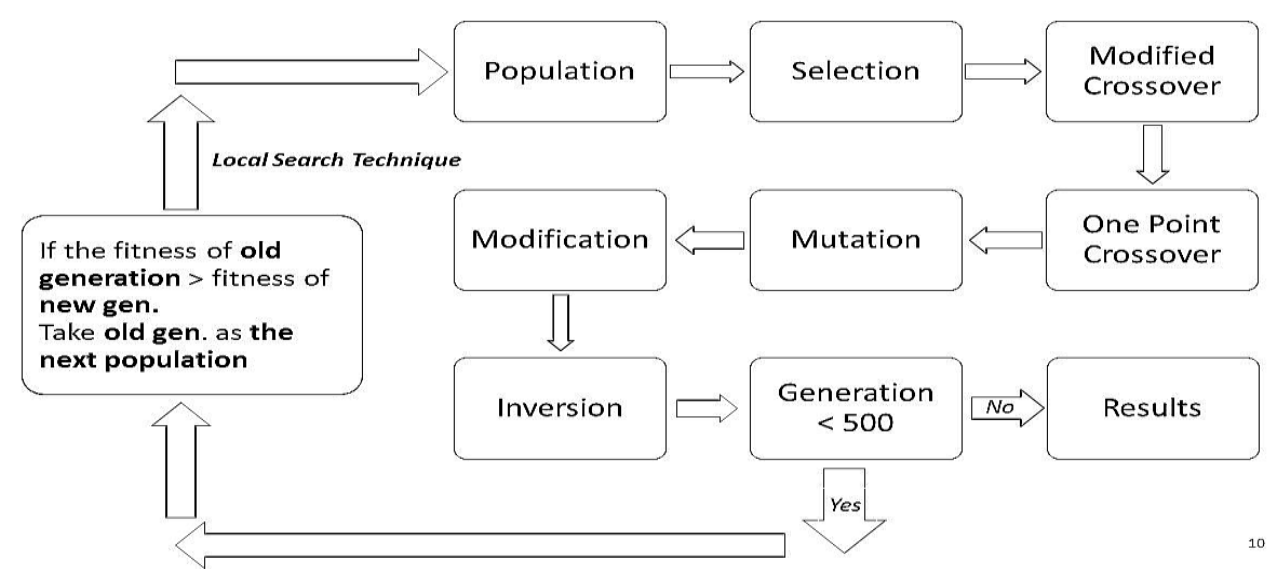

Figure 3. Hybrid algorithm flowchart

\subsection{Results}

\subsubsection{Test Case}

The three algorithms are run for multiple generations on a sample compartment layout shown in Figure 2. Here the distances between compartments are calculated by Dijkstra's algorithm for Improved and Hybrid GA. Also, a set of random values describe the material flow $\mathrm{f}$ and adjacency requirement $\mathrm{c}$ to form an $8 \times 8$ matrix (number of columns/rows in the matrix is the total number of compartments $(08$ in the case of the Sample Layout)) for all the three cases in the objective function mentioned in Equation 2. The values for both matrices range from $0-5$ where 0 signifies no material flow or low adjacency requirement and 5 denotes highest material flow or highest adjacency requirement. The resultant layout computed using the three algorithms considered is tabulated in Table 3.

Table 3. Results from different algorithms

\begin{tabular}{|c|c|}
\hline Method & Layout \\
\hline Islier's Algorithm & 2,4 , passage, $8,1,7$, passage, $3,6,5$ \\
\hline Improved GA & $2,4,1,8$, passage, 3,7 , passage, 5,6 \\
\hline Hybrid GA & $4,2,3,1$, passage, 8,7 , passage, 5,6 \\
\hline
\end{tabular}

Table 4 describes the comparison of different genetic algorithms used. In the table, the results of Basic GA are considered as a baseline to objectively compare the run time and fitness functions of the developed algorithms.

Table 4. Comparative performance of different algorithms

\begin{tabular}{|c|c|c|c|c|}
\hline Method & $\begin{array}{c}\text { Value of } \\
\text { objective } \\
\text { function (F) }\end{array}$ & Run time & Change in F & Change in time \\
\hline Basic GA & 13923.00 & $56.28 \mathrm{~s}$ & $0 \%$ & $0 \%$ \\
\hline Islier's GA & 13802.00 & $54.38 \mathrm{~s}$ & $-0.86 \%$ & $-3.3 \%$ \\
\hline Improved GA & 13745.25 & $35.69 \mathrm{~s}$ & $-1.28 \%$ & $-37 \%$ \\
\hline Hybrid GA & 13567.50 & $100.277 \mathrm{~s}$ & $-2.6 \%$ & $+78 \%$ \\
\hline
\end{tabular}




\subsection{Discussion of Results}

Islier's algorithm performs better than the basic genetic algorithm and succeeds in further minimising the objective function, as shown in Table 4 , along with a reduction in the computational time. It is a good improvement and hence widely used in multi-facility layout problem. However, it does not cater to the movement between compartments through passageways and hence is not suitable for ship compartment layout optimisation.

The improved genetic algorithm succeeds in further minimising the objective function while concluding in lesser time. However, this approach is limited to a case of fixed passageways and does not consider the aspect ratio and area/ location requirements of critical compartments. This limitation is overcome by the hybrid genetic algorithm. However, this function involves a considerably higher computational time. This higher computational time is predominantly attributable to the local search technique (LST), employed to increase the efficiency of arriving at global minima. The plots of the objective functions arrived using all the algorithms mentioned above are shown in Figure 4. The comparison in Table 4 brings out that a hybrid algorithm (using only the local search technique) is a trade-off between accuracy and computational time. Based on computational time alone, the opinion that a hybrid algorithm is slow should not be developed since the approach primarily focuses on improving the efficiency of the result obtained to better assist the designer during the early stages of naval ship layout design and optimisation.
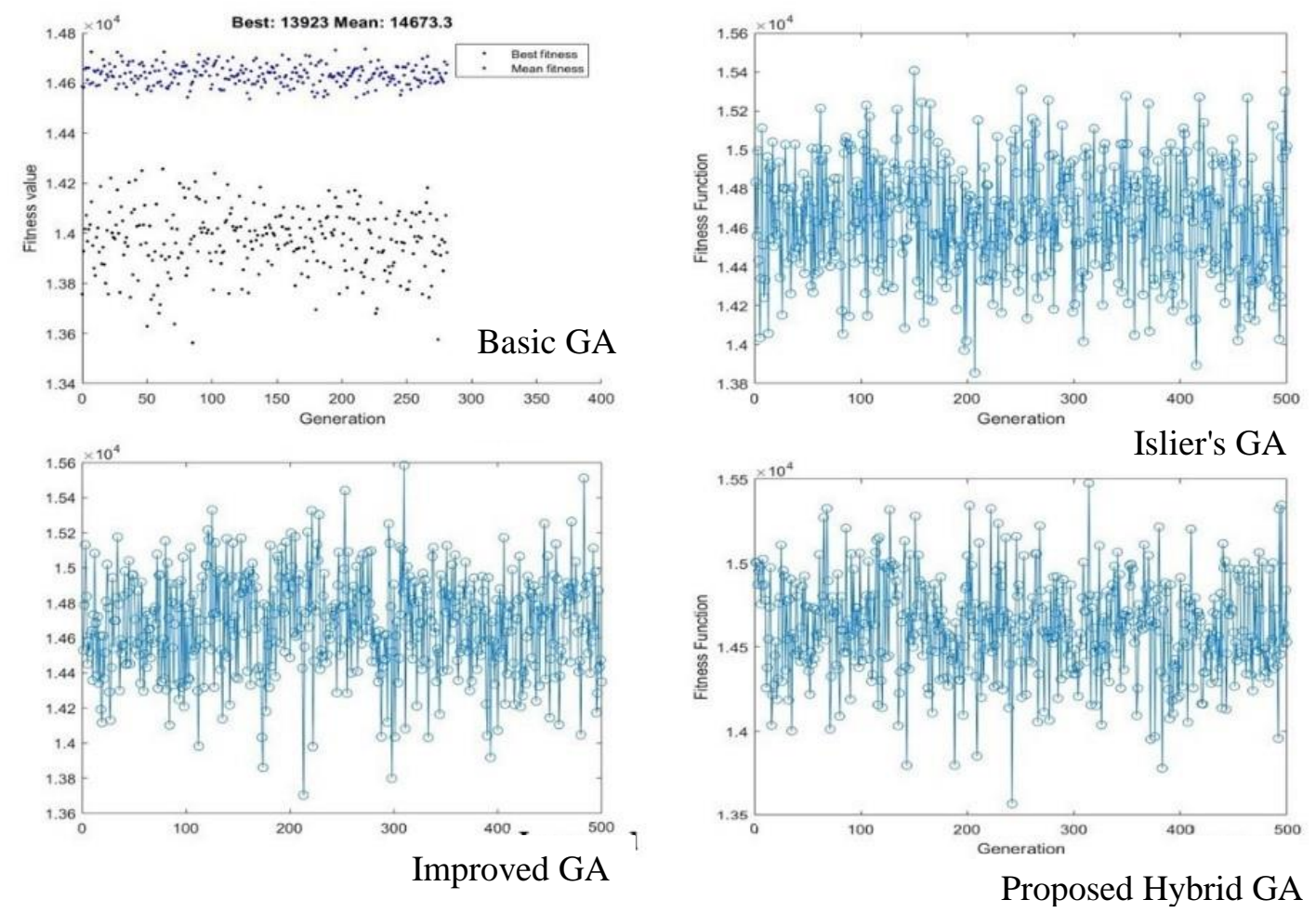

Figure 4. Plots of objective functions obtained using a) basic algorithm, b) Islier's algorithm, c) improved algorithm, and d) hybrid algorithm.

\section{IMPLEMENTATION}

To assess the applicability of Hybrid GA, the authors selected a section between two vertical watertight bulkheads in the mid-region of the second deck of the erstwhile battler ship HMS Battler (Ingalls-Ship-Building-Corporation-Mississippi, 1942). The hybrid GA is applied to optimise the compartment layout of this selection while maintaining the areas of the compartment. The details of the considered ship are: $\mathrm{L}_{\text {overall }}=151.1 \mathrm{~m}$, Beam $=31 \mathrm{~m}$, Draught $=7.1 \mathrm{~m}$, and Displacement $/$ total weight of the ship is 14170 tonnes. The layout of the section considered is shown in Figure 5.

To include the design constraints of the need for individual compartments with fixed locations and adjacency requirements, the Engineer's workshop and the tool issue room are adjacent to each other in all selected options. Figure 6(a) depicts the resultant layout as per the given adjacency and transportation 
weights. The minimised value of objective functions (F) obtained is 17198.32 with a run time of 80.525 seconds. Though the layout is optimised mathematically, a trained naval designer will be able to appreciate that since the position of the engine room in a ship is fixed during the early stages of the design; shifting the engine room casing independently as observed in Fig 6(a) is not practical. This proves the need to improvise the genetic algorithm to ensure the location of functionally critical compartments is not altered during the process of optimization.

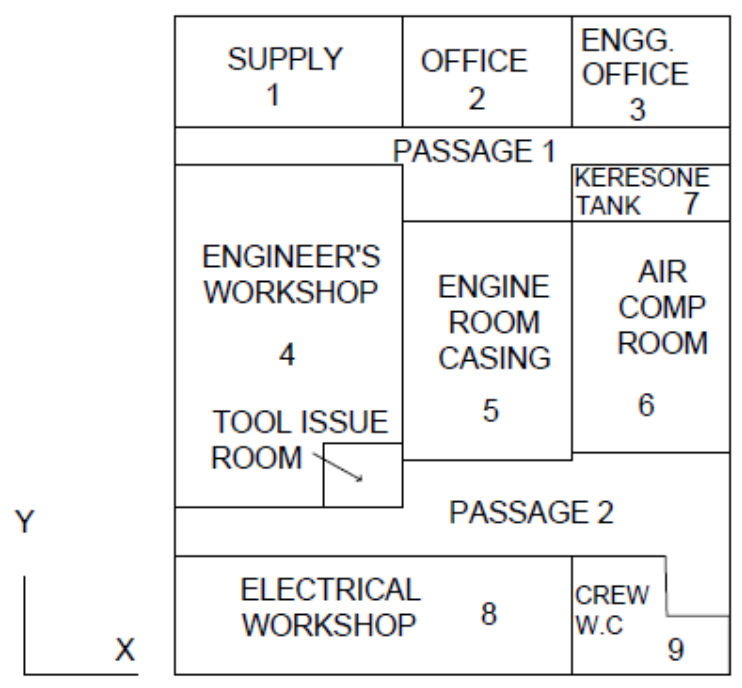

AREAS (sq. meters)

\begin{tabular}{|l|r|}
\hline SUPPLY & 129 \\
OFFICE & 96 \\
ENGG. OFFICE & 88 \\
ENGINEER'S + TOOL ISSUE & 393 \\
WORKSHOP ROOM & \\
ENGINE ROOM CASING & 205 \\
AIR COMP ROOM & 184 \\
KERESONE TANK & 45 \\
ELECTRICAL WORKSHOP & 240 \\
CREW & 75 \\
W.C & \\
\hline
\end{tabular}

Figure 5. Representation of a section deck 2 of HMS Battler (as constructed)
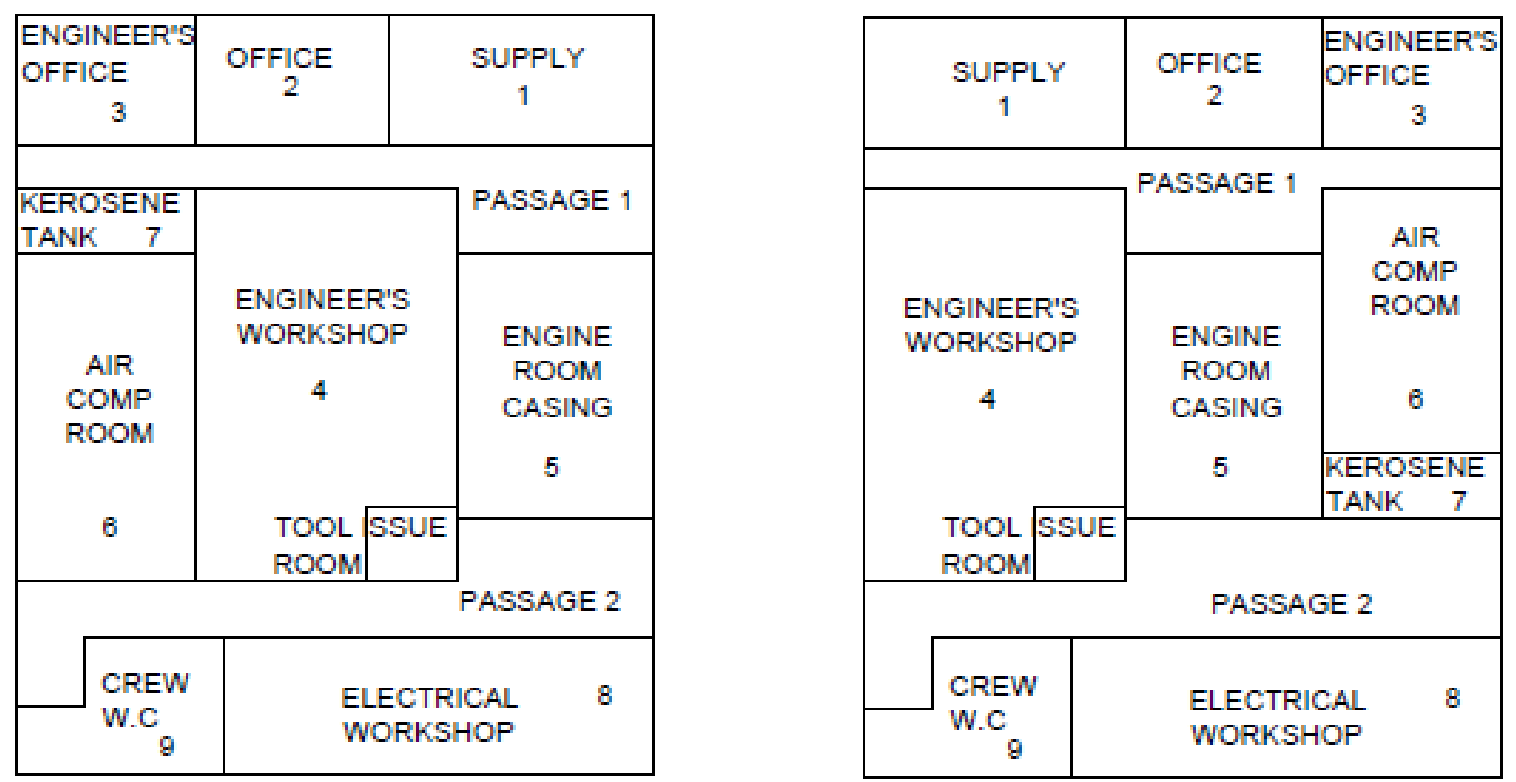

Figure 6. Optimized layout for HMS Battler a) changing engine room location, b) keeping engine room location constant (Post optimization based on distance and adjacency requirements assumed by the author)

The resultant layout arrangement with a fixed location of the engine room is shown in Figure 6(b) where the minimum objective function $(\mathrm{F})$ is 17241.63 in a run time of $70.741 \mathrm{~s}$. Their respective optimization sequences are depicted in Fig 7(a) and 7(b), respectively. 

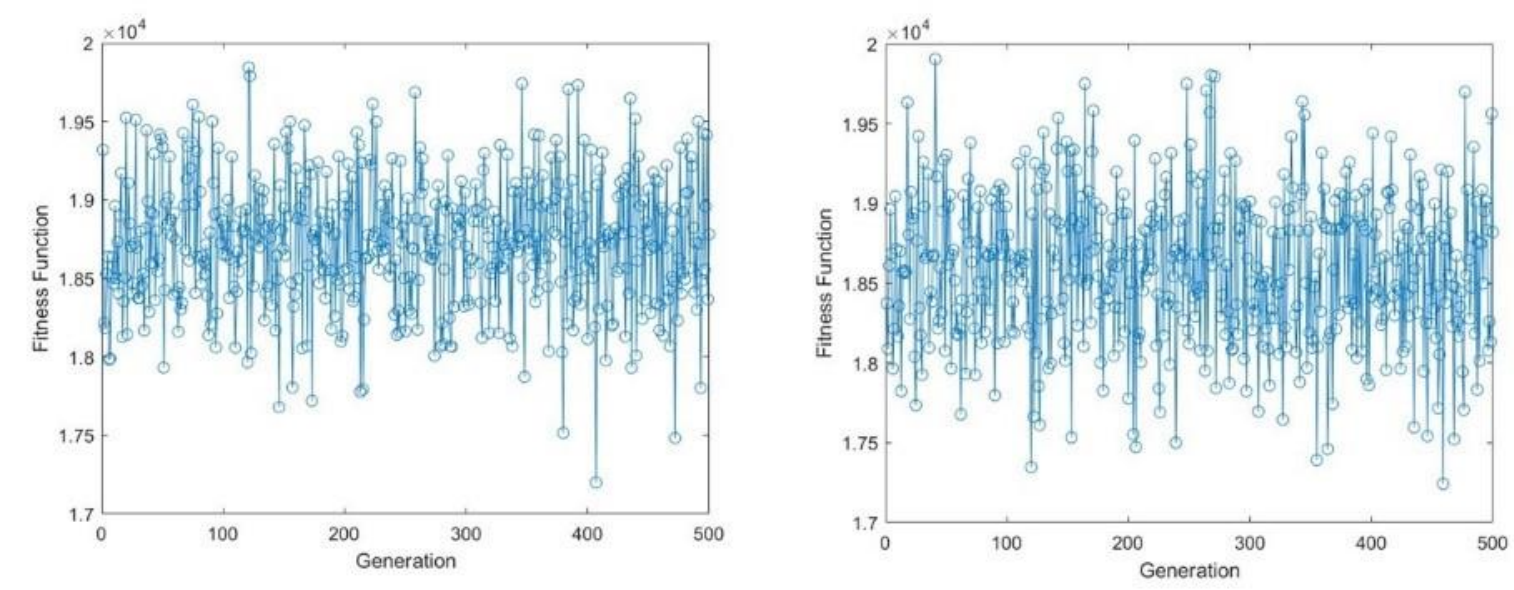

Figure 7. Plots of the objective function for HMS Battler a) changing the position of the engine room, b) keeping the engine room casing constant.

\section{CONCLUSION}

The authors through this research understood the various critical parameters which define the general arrangement of a ship. Therefore, the process of sequential optimization for the development of an optimum general arrangement layout of a naval ship is considered prudent. Further, in the case of final optimization of a single deck, the introduction of design constraints to ensure the position of functionally critical compartments is unchanged was found to be necessary.

The proposed hybrid genetic algorithm is considered to better aid naval designers for the introduction of design constraints as deemed necessary during the progress of the ship design endeavour to develop optimum layouts which are functional. The resultant layout and their corresponding objective functions indicate that though the objective function received from Layout-1 by the improved genetic algorithm is better than that of Layout-2 obtained using the proposed hybrid genetic algorithm. The ver, practical requirements necessitate the finalisation of Layout-2. The improved genetic algorithm is considered to ensure considerable impetus exists on the overall functionality of the ship and the need for specific placement of individual compartments.

Though the authors have primarily focused on the optimisation of naval ship layouts, the concept of sequential optimisation, implementation of Dijkstra's algorithm in measuring distance travelled and catering for the functional constraints of certain compartments in addition to simple mathematical optimisation are considered applicable in a wide range of functions including facility layouts of large complexes, townships and communities which often tend to have multiple objective limitations.

\section{DISCLAIMER}

The contents of this paper are personal views of the author's and does not reflect the official policy of the Indian Navy.

\section{REFERENCES}

Barrera, J., 2020. dijstra very simple, s.l.: MATLAB Central File Exchange.

Chatterjee, S., Carrera, C. \& Lynch, L. A., 1995. Genetic algorithms and travelling salesman problems. European Journal of Operations Research, Volume 1996, pp. 490-510.

Coit, W. D., Smith, A. E. \& Tate, D. M., 1995. Adaptive penalty methods for genetic optimization of constrained combinatorial problem. ORSA Journal on Computing, Volume 1995, p. 31.

Gillespie, J., 2012. A network science approach to understanding and generating ship arrangement in early-stage design, s.l.: University of Michigan.

Hussain, A. et al., 2017a. Genetic algorithm for travelling salesman problem with modified cycle crossover operator. Computer Intelligence and Neuroscience, Volume 2017, p. 7.

Abid Hussain, Yousaf Shad Muhammad, M. Nauman Sajid, Ijaz Hussain, Alaa Mohamd Shoukry, Showkat Gani, "Genetic Algorithm for Traveling Salesman Problem with Modified Cycle Crossover Operator", Computational Intelligence and Neuroscience, vol. 2017b, Article ID 7430125, 7 pages, 2017. https://doi.org/10.1155/2017/7430125 
Igrec, Bojan \& Pawling, Rachel \& Sobey, Adam \& Rigby, Jake \& Thomas, Giles. (2019). An interactive layout exploration and optimisation method for early-stage ship design.

Ingalls-Ship-Building-Corporation-Mississippi, 1942. General arrangement HMS Battler, s.1.: Plan No. AVGP9-01.

Islier, A. A., 1998. A genetic algorithm approach for multiple criteria facility layout design. International Journal of Production Research.

Lee, K. Y., Han, S. N. \& Roh, M. I., 2002. Optimal compartment layout design for a naval ship using an improved genetic algorithm. Marine Technology, Volume 39, pp. 159-167.

Parsons, Michael \& Chung, Hyun \& Kirtley, Eleanor \& Daniels, Anthony \& LIU, SU \& PATEL, JIGNESH. (2008). Intelligent Ship Arrangements: A New Approach to General Arrangement. Naval Engineers Journal. 120. 51 - 65. 10.1111/j.1559-3584.2008.00153. x.

Smith, A. E. \& Coit, D. W., 1997. Constraint-handling techniques- penalty functions. Handbook of Evolutionary Computation, Volume Section C5.2, p. 11.

Wan, W. \& Birch, J. B., 2013. An improved hybrid genetic algorithm with a new local search procedure. Journal of Applied Mathematics, Volume 2013.

Watson, D. G., 1998. Practical ship design. vol 1 ed. s.l. Elsevier. 